\title{
Increase in the Arterial Velocity Pulse Index of Patients with Peripheral Artery Disease
}

\author{
Naotaka Murata Kazuki Shiina Jun Yamashita Nobuhiro Tanaka \\ Taishiro Chikamori Akira Yamashina Hirofumi Tomiyama \\ Department of Cardiology, Tokyo Medical University, Tokyo, Japan
}

\section{Keywords}

Peripheral artery disease $\cdot$ Central hemodynamics $\cdot$ Pressure wave reflection $\cdot$ Arterial velocity pulse index

\begin{abstract}
Background: Recently, a simple parameter calculated from the brachial pressure waveform recorded using an oscillometric device (arterial velocity pulse index [AVI]: ratio of the forward/ reflected pressure wave amplitudes) has become available to assess the pathophysiological abnormalities associated with vascular damage. Peripheral artery disease (PAD) represents one of the disease entities associated with the advanced stages of atherosclerotic vascular damage. The present study was conducted to examine whether an increase in the AVI might be influenced by the presence of PAD. Methods and Results: The AVI was measured from oscillometric recordings of the brachial pressure waveform, and the ankle-brachial pressure index (ABPI) was determined by an oscillometric method. Study 1: In 341 consecutive patients admitted for the management of cardiovascular disease and/or cardiovascular risk factors, the ABPI and the AVI were measured simultaneously. An ABPI $\leq 0.90$ was observed in 19 subjects, and logistic regression analysis revealed a significant association between AVI and ABPI $\leq 0.90$ (odds ratio $=1.81 ; 95 \%$ confidence interval $=1.15-2.84 ; p=0.01$ ). Study 2: In another 19 patients with PAD, percutaneous transluminal angioplasty resulted in a decrease in the AVI from $31 \pm 8$ to $27 \pm 8$ ( $p<0.01)$. Conclusion: Possible presence of PAD must be taken into account while applying the AVI for the assessment of vascular damage.


Murata et al.: Increase in the AVI of Patients with Peripheral Artery Disease

\section{Introduction}

Risk factors for cardiovascular disease, such as hypertension, dyslipidemia, diabetes mellitus, and so on, are encountered commonly in clinical practice. Therefore, simple markers of vascular damage to identify subjects at high risk for cardiovascular disease are needed [1-3]. Recently, the arterial pressure-volume index (API) and the arterial velocity pulse index (AVI), which are obtained by analysis of the brachial pulse pressure waveforms recorded with a regular brachial oscillometric blood pressure cuff, have been introduced as useful markers for clinical settings [4-7]. However, the usefulness of the AVI/API in the screening of subjects with advanced vascular damage has not yet been clarified in detail. Peripheral artery disease (PAD) is one of the manifestations of conditions associated with advanced atherosclerotic vascular damage, but some patients with PAD are asymptomatic. While some markers of vascular damage have been reported to be associated with PAD [8, 9], further study is needed to establish a tool to identify subjects with asymptomatic PAD.

The present study was conducted to examine whether PAD (i.e., subjects with an anklebrachial pressure index $[\mathrm{ABPI}] \leq 0.90$ ) is an independent factor associated with an increase in the AVI/API.

\section{Methods}

Study Participants and Study Design

Study 1: Examination of the Association of the AVI/API with PAD. The subjects of this study were the participants of a previous study [5]. In brief, they consisted of 352 consecutive patients hospitalized at the Department of Cardiology of the Tokyo Medical University Hospital for the management of their cardiovascular disease/cardiovascular risk factors (e.g., coronary heart disease, arrhythmia, sleep apnea, etc.). Patients admitted for the management of cardiac emergencies, such as acute coronary syndrome, acute exacerbation of heart failure, infectious disease, etc. were excluded from the study. On the day of admission, the subjects were asked to rest for at least 15 min before first undergoing measurement of the AVI/API, followed by measurement of the ABPI. The AVI/API and ABPI measurements were conducted in the afternoon, and the patients were requested to avoid intake of food within at least $2 \mathrm{~h}$ prior to the measurements.

Study 2: Examination of the Effect of Endovascular Therapy on the API/AVI. Measurements of the AVI/ API were performed before and after endovascular therapy (EVT) undertaken for the treatment of lower limb arterial stenosis in 19 consecutive patients with PAD admitted to the Tokyo Medical University Hospital (they were different patients from study 1 ).

\section{AVI/API Measurements}

API, AVI, systolic blood pressure, diastolic blood pressure, pulse pressure, and heart rate were measured simultaneously with the brachial pulse waveforms recorded with the subjects in the seated position using a regular brachial oscillometric blood pressure cuff (Pasesa AVE-1500; Shisei Datum, Yokohama, Japan). The details of the measurements of these parameters are described elsewhere [4-6]. Briefly, a conventional blood pressure cuff was wrapped around the left upper arm and inflated steadily at the rate of $10 \mathrm{~mm} \mathrm{Hg} / \mathrm{s}$, up to $190 \mathrm{~mm} \mathrm{Hg}$, and then the cuff was deflated to $10 \mathrm{~mm} \mathrm{Hg}$ at the rate of $3 \mathrm{~mm} \mathrm{Hg} / \mathrm{s}$. Cuff pressures during inflation and deflation, measured using a pressure transducer, were stored. Using the amplitudes of all the pulse oscillations and changes in the cuff pressure for pulse pressure from the pressure point evoked by the pulses, we calculated the local slopes of the curve between the cuff pressure and the arterial volume. We calculated the numerical integration of the averaged slopes to generate pressure-volume curves and identified the numerical coefficient of the equation as the API. Thus, the API is thought to be a marker of the local arterial stiffness. It reflects the characteristics of the pulse waveform when the cuff pressure is higher than the maximum blood pressure. Based on the cuff pressure waveforms, the differentiated waveforms of the forward pressure wave (Vf) and reflected pressure wave (Vr) were obtained. The AVI was calculated as $20 \times \mathrm{Vr} / \mathrm{Vf}$. Thus, the AVI is thought to be a marker of pressure wave reflection. 
Murata et al.: Increase in the AVI of Patients with Peripheral Artery Disease

ABPI Measurement

The ABPI was measured using a volume-plethysmographic apparatus (Form/ABI; Omron Health Care Co., Ltd., Kyoto, Japan), in accordance with a previously described methodology [10]. In brief, occlusion cuffs connected to plethysmographic and oscillometric sensors were tied around both the upper arms and lower legs with the subjects lying in the supine position. The brachial and post-tibial arterial pressures were measured using the oscillometric sensor.

Laboratory Measurements

The serum levels of low-density lipoprotein cholesterol, high-density lipoprotein cholesterol, triglyceride, creatinine, and plasma hemoglobin A1c were measured enzymatically.

\section{Statistical Analysis}

Data are expressed as means \pm standard deviations. Categorical data are expressed as percentages. For assessment of the differences in the status of each variable between the groups, the Student $t$ test or the $\chi^{2}$ test was applied. The changes in AVI and API after the EVT as compared to the values prior to the intervention were assessed by a paired $t$ test. The relationship of the AVI and API with ABPI $\leq 0.90$ was examined by logistic regression analysis with/without adjustments. All of the analyses were conducted using the IBM/ SPSS software for Windows, version 23.0J (IBM/SPSS Inc., Chicago, IL, USA); $p$ values $<0.05$ were considered as denoting statistical significance.

\section{Results}

In study 1 (examination of the association of the AVI with PAD), among the 352 consecutive study subjects, 11 subjects (including 5 with atrial fibrillation and 6 with left ventricular ejection fraction values $<40 \%$ ) were excluded from the analyses, and the remaining 341 subjects were included. The ABPI was $\leq 0.90$ in 19 patients. The clinical characteristics of the participants of study 1 are shown in Table 1 . Both the AVI and the API were higher in subjects with an ABPI $\leq 0.90$ than in those with an ABPI $>0.90$. The results of the logistic regression analysis revealed that the odds ratio of the AVI for identification of subjects with an ABPI $\leq 0.90$ was significant, even after adjustments for confounding variables (age, sex, height, mean blood pressure, and heart rate), and that of the API was only marginally significant (Table 2). When the AVI and the API were entered simultaneously in the same model, it was the AVI rather than the API that was significantly associated with ABPI $\leq 0.90$ (Table 2).

The clinical characteristics of the participants of study 2 (examination of the effect of EVT on the API/AVI) are shown in Table 3. Both the AVI and the API were significantly higher in the subjects of study 2 as compared to the values in the subjects of study 1 with ABPI $>0.90$ $(p<0.01)$. EVT for lower limb arterial stenosis was successfully accomplished in all of the patients with PAD, following which the AVI decreased and the ABPI increased significantly in all patients (Fig. 1).

\section{Discussion}

The novelty of the present study is that both the AVI and the API were higher in subjects with $\mathrm{ABPI} \leq 0.90$, that it was the AVI rather than the API that was significantly associated with $\mathrm{ABPI} \leq 0.90$, and that improvement of lower limb arterial stenosis by EVT resulted in a decrease in the AVI in subjects with PAD.

The API and the AVI, which are obtained by pressure waveform analysis of the brachial pulse waveforms recorded using a regular brachial oscillometric blood pressure cuff, have become available for use in clinical settings [4-7]. Recently, we reported that the AVI was higher in patients admitted for coronary angiography (i.e., subjects suspected to have 
Table 1. Clinical characteristics of the subjects in study 1 (examination of the association of the arterial velocity pulse index with peripheral artery disease)

\begin{tabular}{lccc}
\hline & $\begin{array}{c}\text { ABPI }>0.90 \\
(n=322)\end{array}$ & $\begin{array}{c}\text { ABPI } \leq 0.90 \\
(n=19)\end{array}$ & $p$ value \\
\hline Age, years & $60 \pm 14$ & $71 \pm 14$ & 0.002 \\
Men/women (\%) & $248 / 74(77 \%)$ & $16 / 3(84 \%)$ & 0.462 \\
Body mass index & $25.4 \pm 4.5$ & $23.5 \pm 3.3$ & 0.055 \\
Current smokers & $51(16 \%)$ & $2(10 \%)$ & 0.531 \\
Systolic blood pressure, mm Hg & $128 \pm 18$ & $138 \pm 27$ & 0.008 \\
Diastolic blood pressure, mm Hg & $73 \pm 12$ & $68 \pm 14$ & 0.095 \\
Heart rate, beats/min & $69 \pm 13$ & $68 \pm 8$ & 0.572 \\
Ankle-brachial index & $1.16 \pm 0.09$ & $0.75 \pm 0.13$ & $<0.001$ \\
Arterial velocity pulse index & $23 \pm 9$ & $33 \pm 10$ & $<0.001$ \\
Arterial pressure-volume index & $25 \pm 6$ & $30 \pm 6$ & $<0.001$ \\
Serum HDL-C, mmol/L & $1.3 \pm 0.4$ & $1.5 \pm 0.4$ & 0.183 \\
Serum triglyceride, mmol/L & $1.8 \pm 1.1$ & $1.6 \pm 0.8$ & 0.602 \\
Serum LDL-C, mmol/L & $2.8 \pm 0.7$ & $2.2 \pm 0.7$ & 0.018 \\
Hemoglobin A1c, \% & $5.6 \pm 0.7$ & $6.1 \pm 1.0$ & 0.031 \\
Serum creatinine, $\mu$ mol/L & $81 \pm 28$ & $73 \pm 19$ & 0.285 \\
Med HBP & $180(56 \%)$ & $10(53 \%)$ & 0.769 \\
Med DM & $66(21 \%)$ & $5(26 \%)$ & 0.549 \\
Med DLIP & $181(56 \%)$ & $6(32 \%)$ & 0.035 \\
\hline
\end{tabular}

Values are presented as mean \pm standard deviation or $n(\%)$. ABPI, ankle-brachial pressure index; API, arterial pressure-volume index; HDL-C, high-density lipoprotein cholesterol; LDL-C, low-density lipoprotein cholesterol; Med DLIP, subjects receiving medication for dyslipidemia; Med DM, subjects receiving medication for diabetes mellitus; Med HBP, subjects receiving medication for hypertension.

Table 2. Results of logistic regression analysis to examine the relationship of the AVI and the API with ABPI $\leq 0.90$ (outcome variable $=\mathrm{ABPI} \leq 0.90$ )

\begin{tabular}{lccc}
\hline Explanatory & $\begin{array}{l}\text { Odds } \\
\text { ratio }\end{array}$ & $\begin{array}{l}\text { 95\% confidence } \\
\text { interval }\end{array}$ & $p$ value \\
\hline Crude & & & \\
AVI (per SD increase) & 2.10 & $1.78-2.98$ & $<0.01$ \\
API (per SD increase) & 1.69 & $1.15-2.47$ & $<0.01$ \\
\hline Adjusted & & & \\
AVI (per SD increase) & 1.84 & $1.18-2.87$ & $<0.01$ \\
API (per SD increase) & 1.64 & $0.99-2.71$ & 0.06 \\
\hline Adjusted: AVI and API entered simultaneously in the same model \\
AVI (per SD increase) & 1.81 & $1.15-2.84$ & 0.01 \\
API (per SD increase) & 1.61 & $0.95-2.73$ & 0.08 \\
\hline
\end{tabular}

Adjusted for age, sex, height, mean blood pressure, and heart rate. API, arterial pressure-volume index; AVI, arterial velocity pulse index; $\mathrm{SD}$, standard deviation.

advanced atherosclerosis, e.g., coronary artery disease) than in those admitted for other reasons than coronary angiography (i.e., subjects not suspected to have advanced atherosclerosis, e.g., arrhythmia) [5]. Hitsumoto [6] reported that the AVI was significantly higher in patients with detectable high-sensitivity troponin $\mathrm{T}$ than in those without detectable highsensitivity troponin T. Sasaki-Nakashima et al. [7] reported that the API was significantly and 


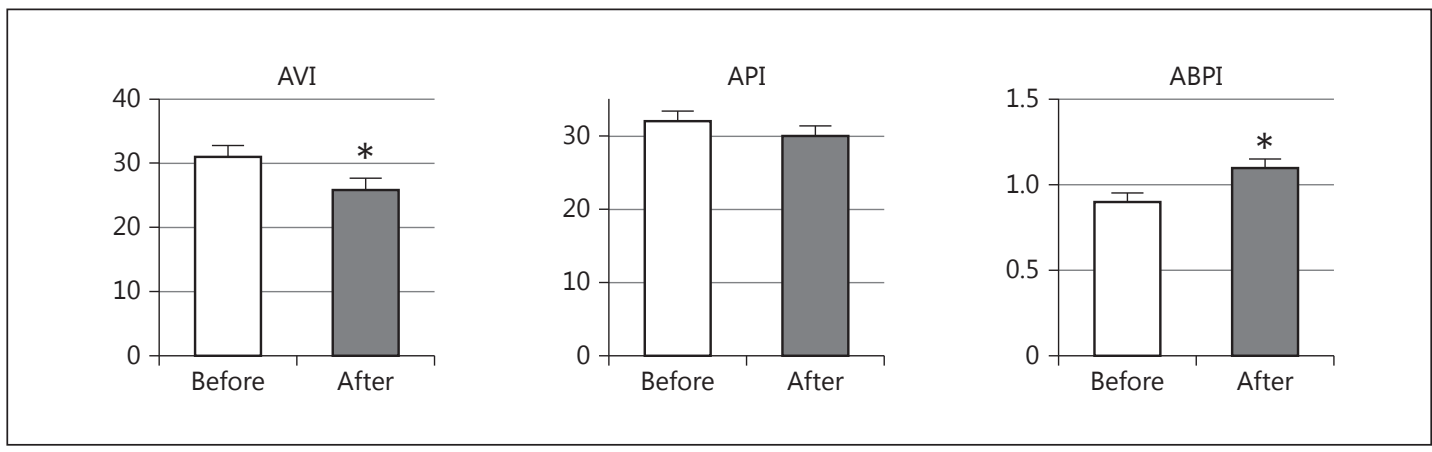

Fig. 1. Changes in the variables after endovascular therapy. ${ }^{*} p<0.05$ versus before endovascular therapy. AVI, arterial velocity pulse index; API, arterial pressure-volume index; ABPI, ankle-brachial pressure index.

Table 3. Clinical characteristics of the subjects in study 2 (examination of the effect of endovascular therapy on the arterial velocity pulse index/ arterial pressure-volume index)

$\begin{array}{lc}\text { Age, years } & 69 \pm 9 \\ \text { Men/women } & 14 / 5(73 \%) \\ \text { Body mass index } & 23.3 \pm 2.6 \\ \text { Current smokers } & 4(22 \%) \\ \text { Systolic blood pressure, mm Hg } & 121 \pm 23 \\ \text { Diastolic blood pressure, mm Hg } & 70 \pm 14 \\ \text { Heart rate, beats/min } & 76 \pm 13 \\ \text { Ankle-brachial index } & 0.88 \pm 0.22 \\ \text { Arterial velocity pulse index } & 31 \pm 8 \\ \text { Arterial pressure-volume index } & 32 \pm 6 \\ \text { Serum HDL-C, mmol/L } & 1.3 \pm 0.4 \\ \text { Serum triglyceride, mmol/L } & 1.9 \pm 1.5 \\ \text { Serum LDL-C, mmol/L } & 2.6 \pm 0.66 \\ \text { Hemoglobin A1c, \% } & 6.8 \pm 1.1 \\ \text { Serum creatinine, } \mu \text { mol/L } & 88 \pm 31 \\ \text { Med HBP } & 16(84 \%) \\ \text { Med DM } & 13(68 \%) \\ \text { Med DLIP } & 10(42 \%)\end{array}$

Values are presented as mean \pm standard deviation or $n(\%)$. HDL-C, high-density lipoprotein cholesterol; LDL-C, low-density lipoprotein cholesterol; Med DLIP, subjects receiving medication for dyslipidemia; Med DM, subjects receiving medication for diabetes mellitus; Med HBP, subjects receiving medication for hypertension.

independently associated with both the Framingham Cardiovascular Risk Score and the Suita Score. In the present study, both the AVI and the API were higher in subjects with an ABPI $\leq 0.90$. Thus, because of the advanced sclerotic vascular damage in subjects with PAD, the API and the AVI may be simple-to-measure markers reflecting the pathophysiological abnormalities related to sclerotic vascular damage (i.e., arteriosclerosis and/or atherosclerosis).

The ABPI, which is usually measured by the Doppler method or the oscillometric method, is widely accepted as a marker for PAD screening [11]. The list of indications for ABPI measurement and the number of candidates for the measurement of this parameter are large [11]. However, measurement by the Doppler method requires a high level of skill $[12,13]$, and measurement by the oscillometric method requires a dedicated equipment [10]. Therefore, 
ABPI measurement has not been implemented in all potential candidates for measurement of this parameter. An alternative approach for screening to detect asymptomatic PAD is to apply a simpler method, if possible, for PAD screening. In the present study, the results of logistic regression analysis revealed that the odds ratio of the AVI for identification of subjects with an ABPI $\leq 0.90$ was significant. Further study is proposed to examine the usefulness of the AVI for the screening of subjects for PAD.

The speculative explanation for the association of increased AVI with the presence of PAD is as follows: The augmentation index (AI) reflects the summation of the forward pressure wave and reflected pressure wave in the arterial tree [1,2]. Several studies have demonstrated an elevation of the $\mathrm{AI}$ in subjects with PAD, in other words, an inverse correlation between the ABPI and the AI $[8,9]$. A plausible explanation for this finding is that in the arterial tree, pressure wave reflection occurs at the site of increased arterial resistance, and arterial narrowing/severe stenosis is associated with a steep increase in arterial resistance. The AI reflects the summation of the forward pressure wave and the reflected pressure wave $[1,2]$, and the AVI is thought to be a marker of the ratio of the amplitude of the forward pressure wave to that of the reflected pressure wave [4,5]. Therefore, the AVI may also be affected by abnormalities of the reflected pressure wave associated with arterial narrowing/ severe stenosis. Jacomella et al. [14] have already demonstrated that successful EVT for lower limb arterial stenosis reduces the AI. In the present study, EVT was followed by a reduction in the AVI as well. Therefore the pressure wave reflection, which may occur prematurely because of arterial narrowing/severe stenosis, might increase the AVI. Further study is needed to confirm this speculative explanation.

The limitation of this study is that it was conducted in subjects with cardiovascular disease and/or risk factors for cardiovascular disease. Age and blood pressure affect the AVI [5]. While age and blood pressure were included as covariates in the analysis model used in this study for adjustments, the present findings need to be confirmed in the elderly, in subjects with hypertension, and also in the general population.

\section{Conclusion}

The presence of underlying PAD must be taken into account during the assessment of vascular damage based on the AVI.

\section{Statement of Ethics}

The studies were conducted with the approval of the Ethics Committee of Tokyo Medical University. Written informed consent was obtained from each subject before he/she was enrolled in the study.

\section{Disclosure Statement}

For this study, a Pasesa AVE-1500 (Shisei Datum, Yokohama, Japan), which is used to measure the API and the AVI, was leased free of charge from Nihon Koden Company, Tokyo, Japan. This research received no grant from any funding agency in the public, commercial, or not-for-profit sectors. 


\section{References}

1 Tomiyama H, Yamashina A: Non-invasive vascular function tests: their pathophysiological background and clinical application. Circ J 2010;74:24-33.

-2 O'Rourke MF, Hashimoto J: Mechanical factors in arterial aging: a clinical perspective. J Am Coll Cardiol 2007; 50:1-13.

-3 Niiranen TJ, Vasan RS: Epidemiology of cardiovascular disease: recent novel outlooks on risk factors and clinical approaches. Expert Rev Cardiovasc Ther 2016;14:855-869.

4 Komine H, Asai Y, Yokoi T, Yoshizawa M: Non-invasive assessment of arterial stiffness using oscillometric blood pressure measurement. Biomed Eng Online 2012;11:6.

5 Komatsu S, Tomiyama H, Kimura K, Matsumoto C, Shiina K, Yamashina A: Comparison of the clinical significance of single cuff-based arterial stiffness parameters with that of the commonly used parameters. J Cardiol 2017;69:678-683.

6 Hitsumoto T: Arterial velocity pulse index as a novel marker of atherosclerosis using pulse wave analysis on high sensitivity troponin T in hypertensive patients. Cardiol Res 2017;8:36-43.

-7 Sasaki-Nakashima R, Kino T, Chen L, Doi H, Minegishi S, Abe K, Sugano T, Taguri M, Ishigami T: Successful prediction of cardiovascular risk by new non-invasive vascular indexes using suprasystolic cuff oscillometric waveform analysis. J Cardiol 2017;69:30-37.

-8 Paapstel K, Zilmer M, Eha J, Tootsi K, Piir A, Kals J: Association between fibulin-1 and aortic augmentation index in male patients with peripheral arterial disease. Eur J Vasc Endovasc Surg 2016;51:76-82.

-9 Mosimann K, Jacomella V, Thalhammer C, Meier TO, Kohler M, Amann-Vesti B, Husmann M: Severity of peripheral arterial disease is associated with aortic pressure augmentation and subendocardial viability ratio. J Clin Hypertens (Greenwich) 2012;14:855-860.

10 Yamashina A, Tomiyama H, Takeda K, Tsuda H, Arai T, Hirose K, Koji Y, Hori S, Yamamoto Y: Validity, reproducibility, and clinical significance of noninvasive brachial-ankle pulse wave velocity measurement. Hypertens Res 2002;25:359-364.

11 Aboyans V, Criqui MH, Abraham P, Allison MA, Creager MA, Diehm C, Fowkes FG, Hiatt WR, Jönsson B, Lacroix P, Marin B, McDermott MM, Norgren L, Pande RL, Preux PM, Stoffers HE, Treat-Jacobson D; American Heart Association Council on Peripheral Vascular Disease; Council on Epidemiology and Prevention; Council on Clinical Cardiology; Council on Cardiovascular Nursing; Council on Cardiovascular Radiology and Intervention, and Council on Cardiovascular Surgery and Anesthesia: Measurement and interpretation of the anklebrachial index: a scientific statement from the American Heart Association. Circulation 2012;126:2890-2909.

12 Bendermacher BL, Teijink JA, Willigendael EM, Bartelink ML, Peters RJ, Langenberg M, Büller HR, Prins MH: Applicability of the ankle-brachial-index measurement as screening device for high cardiovascular risk: an observational study. BMC Cardiovasc Disord 2012;12:59.

13 Davies JH, Kenkre J, Williams EM: Current utility of the ankle-brachial index (ABI) in general practice: implications for its use in cardiovascular disease screening. BMC Fam Pract 2014;15:69.

14 Jacomella V, Shenoy A, Mosimann K, Kohler MK, Amann-Vesti B, Husmann M: The impact of endovascular lower-limb revascularisation on the aortic augmentation index in patients with peripheral arterial disease. Eur J Vasc Endovasc Surg 2013;45:497-501. 